\title{
CD99 ligation upregulates HSP70 on acute lymphoblastic leukemia cells and concomitantly increases NK cytotoxicity
}

\author{
Z Husak ${ }^{\star, 1}$ and MN Dworzak ${ }^{*, 1,2}$
}

CD99 is present in many human cell types, including high-level surface expression on pediatric B and T leukemias and Ewing tumors (ETs). On B lymphocytes and respective malignancies, its level decreases with the stage of maturation. Inter-individual variability of CD99 on B-cell precursor acute lymphoblastic leukemia (BCP-ALL) blasts was shown recently to be associated with distinct cytogenetic backgrounds. However, CD99 targets remain mainly unknown. Here, we show that administration of an antiCD99 antibody to B- and T-leukemia cell lines induces heat shock protein 70 (HSP70), both on the cell surface and in the cytoplasm. Investigation of primary BCP-ALL cells rendered similar results. Intriguingly, CD99-induced modulation of HSP70 on ET cells had profiles different from that on leukemia cells. Since HSP70 expression on tumor cells is a prerequisite for natural killer (NK) cell-mediated tumor lysis, we hypothesized that CD99-induced HSP70 may allow targeting of some CD99-positive malignancies via NK-cell cytotoxicity. Our experiments with NK92 cell line demonstrated that leukemia cells with upregulated HSP70 can be successfully killed by effector cells. We consider our data as a new view of CD99 functions and as a basis for the development of a potential anti-tumor strategy based on heat-shock protein activation via CD99 triggering.

Cell Death and Disease (2012) 3, e425; doi:10.1038/cddis.2012.164; published online 15 November 2012

Subject Category: Cancer

CD99 is a $32-k D$ transmembrane protein with a high-level surface expression on pediatric leukemias and Ewing tumor (ET) cells. ${ }^{1,2}$ On B lymphocytes, its level is determined by maturation and is saved at the respective stage of malignancy: ${ }^{3}$ more mature B-cell precursors (BCPs) carry less CD99 on the cell surface. Variability of CD99 on blasts from different BCP-ALL (acute lymphoblastic leukemia) patients is associated with distinct cytogenetic backgrounds, and ETV6/ RUNX1-positive BCP-ALLs were found to be particularly sensitive to CD99 ligation by monoclonal antibodies (mAbs). ${ }^{4}$ Involvement of CD99 in diverse intracellular and extracellular processes (adhesion, migration and apoptosis) was described for lymphocytes and some other cells types. ${ }^{5-7}$ However, signaling pathways triggered by CD99 are not yet completely defined.

Several lines of evidence indicate that CD99 shares some important properties with HSP70 - a member of heat-shock proteins, most conserved protein group in living organisms, abundantly expressed on the constitutive level or upon external influence. ${ }^{8}$ Within B-lineage cells, the constitutively expressed HSP70 family member HSC70 (like CD99) is associated with well-defined differentiation stages. ${ }^{3,9}$ Bone marrow-derived leukemia blasts from patients with different hematological malignancies are frequently HSP70 membrane positive $^{10}$ as well as CD99 positive. ${ }^{1,3}$ In childhood, ALL
HSP70 is connected to the actin cytoskeleton ${ }^{11}$ - and a link of CD99 to actin was found in Ewing sarcoma. ${ }^{12}$ Further, overexpression of HSP70 increases surface levels of MHC class I, ${ }^{13}$ and engagement of CD99 triggers transport of $\mathrm{MHC}$ I from the Golgi complex to the cell surface along with rearrangement of the actin cytoskeleton. ${ }^{14,15}$ Next, CD10 which is a surface marker of BCP and which correlates with CD99 in BCP-ALL ${ }^{4}$ may physically interact with HSP70. ${ }^{16}$ Portions of CD99 as well as HSP70 are found in lipid rafts cell membrane microdomains that serve as interaction platform for highly concentrated proteins. ${ }^{15,17}$ A functional relationship between ETV6/RUNX1-positive ALL and heatshock proteins was found in studies on downregulation of HSP70 after ETV6/RUNX1 depletion ${ }^{18}$ - and mainly ETV6/ RUNX1-positive blasts were found to be affected by CD99 ligation. ${ }^{4}$ Finally, as CD99 was shown to be an upregulated target of the von Hippel-Lindau/hypoxia pathway ${ }^{19}$ and HSP70 was found to be involved in inhibition of oxidative stress-mediated apoptosis (reviewed in ref. 20), both proteins seem to be implicated in oxygen deregulation processes. All these facts suggest functional links between CD99 and HSP70. We describe here a novel signaling pathway where CD99 modulates expression of HSP70. Since HSP70 promotes natural killer (NK)-cell activity against tumors, ${ }^{21}$ we examined targeting of some CD99-positive malignancies -

\footnotetext{
1Department of Immunological Diagnostics, St. Anna Kinderkrebsforschung, Children's Cancer Research Institute, Vienna, Austria and ${ }^{2}$ Hemato-oncology Station, St. Anna Kinderspital, Vienna, Austria

${ }^{*}$ Corresponding authors: Z Husak, Department of Immunological Diagnostics, St. Anna Kinderkrebsforschung, Children's Cancer Research Institute, Zimmermannplatz 10, 1090 Vienna, Austria. Tel: +43 140470 4065; Fax: +43 140470 7150; E-mail: zvenyslava.husak@ ccri.at

or MN Dworzak, Hemato-oncology Station, St. Anna Kinderspital, Kinderspitalgasse 6, 1090 Vienna, Austria. Tel: +43 1404704064 ; Fax: +43 14047064064 ; E-mail: dworzak@stanna.at

Keywords: CD99; HSP70; leukemia; cytotoxicity

Abbreviation: NK, natural killer cells

Received 02.5.12; revised 24.9.12; accepted 27.9.12; Edited by G Ciliberto
} 
B and T ALLs, ETs - by cytotoxic NK92 cell line. ${ }^{22}$ Our findings demonstrate that CD99 ligation on leukemia cells is effective tool to increase NK-cell activity toward targets and this process correlates with upregulation of HSP70 on the leukemia cell surfaces.

\section{Results}

CD99-induced HSP70 expression in B and T Iymphocytes. Based on a potential interaction of CD99 and HSP70, we decided to check expression levels of HSP70 after CD99 engagement with specific mAb (DN16, hec2 and O662). Our first experiments with Reh (ETV6/RUNX1-positive BCP-ALL cell line) showed that CD99 ligation strongly (up to 3-fold) and rapidly (within $3 \mathrm{~h}$ ) upregulated HSP70 in the cytoplasm (cy) (Figure 1a) and on the cell surface (s) (Figure 1b) - a dynamics and strength compatible with a heat shock influence. ${ }^{23}$ The response reached its maximum at day 3 of incubation with anti-CD99 antibody. We found CD99-induced upregulation of HSP70 levels in all conducted experiments. To test the specificity of HSP70 response, Reh cells were incubated with antibodies against CD10 and CD19 differentiation markers of BCPs. Levels of (s)HSP70 were not modulated via these antigens (Figure 1b). Also incubation of cell lines Raji (CD99 dim/neg mature human B cells) and El4 (CD99 ${ }^{\text {neg }}$ mouse $T$ cells) with DN16 did not influence their (s)HSP70 expression. In contrast, CD99 highly expressing Jurkat cells (T-ALL) responded with 3- to 7-fold maintenance of HSP70 (Figure 1b).

Analysis of 21 samples from BCP-ALL patients with different cytogenetic backgrounds (six with hyperdiploidy, six with ETV6/RUNX1 rearrangement and nine with random karyotype) showed HSP70 in each case to be expressed at a higher level in cytoplasm than on the surface (ratio of cy/s $\mathrm{HSP} 70=$ from 2 to 3 ) with significant correlation between cytoplasmatic and surface forms $(P<0.01, r=0.79)$ (Figure 1c).
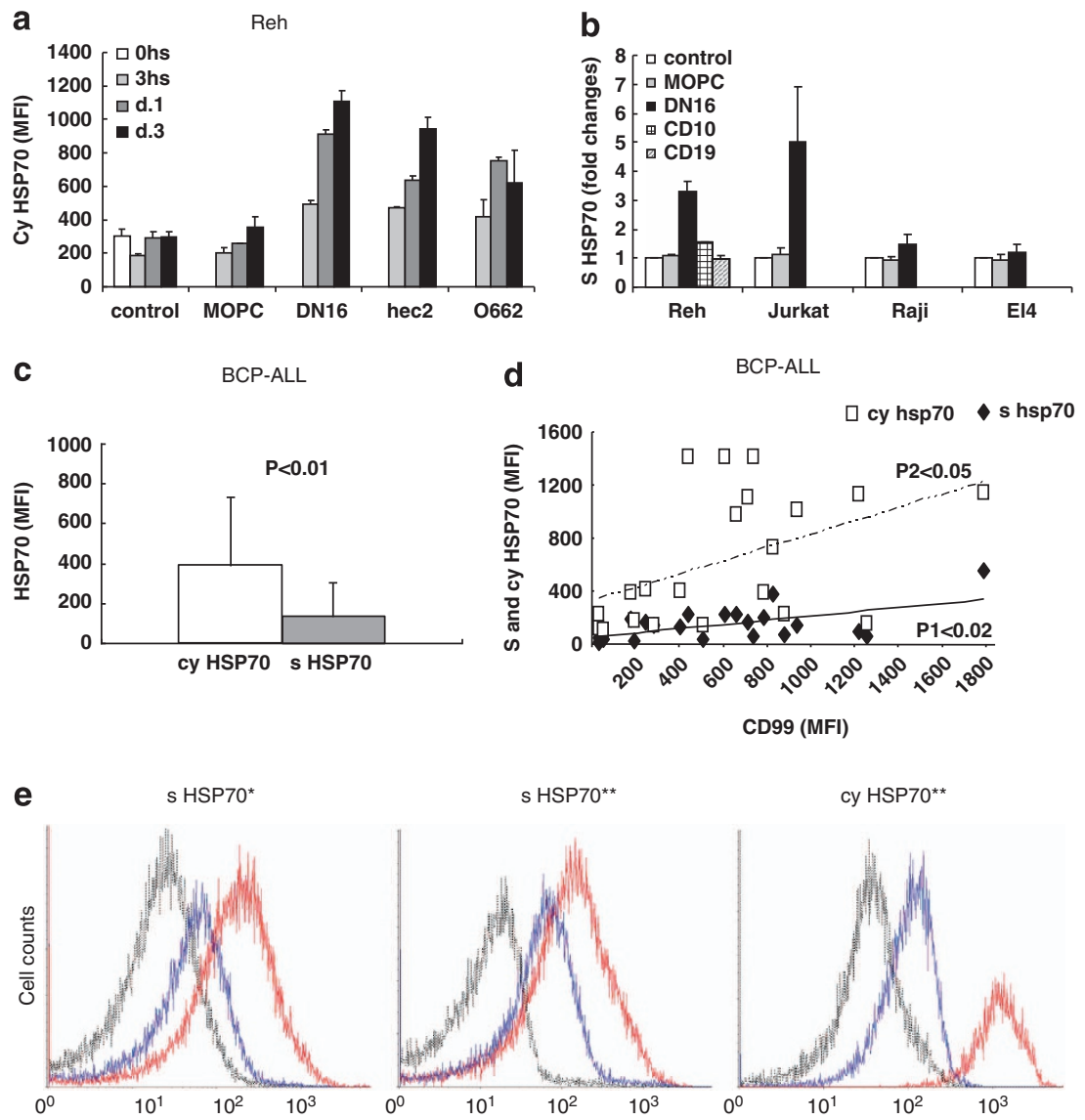

Figure 1 CD99 ligation increases HSP70 expression in CD99-positive human B and T lymphocytes. (a) Time-dependent upregulation of cytoplasmatic (cy) HSP70 in Reh cells: cells were cultured for the indicated time in the presence of $10 \mu \mathrm{g} / \mathrm{ml}$ of CD99 mAbs (DN16, O662 and hec2), MOPC1 (control mAb) or without treatment (control). Annexin V and 7AAD were used to stain apoptotic and dead cells. In all performed experiments, HSP70 was measured on non-apoptotic cells. Error bars indicate S.E.M. of triplicate wells. Data represent six independent experiments. (b) HSP70 upregulation is a specific response upon CD99 ligation in Reh cells: cells were treated with the indicated mAbs for $3 \mathrm{~h}$. Strong surface (s) HSP70 upregulation was observed in CD99-highly expressing Reh and Jurkat cell lines. CD99-low expressing Raji and CD99negative murine EI4 cells did not exhibit HSP70 response when incubated with DN16. Error bars show S.E.M. of three independent experiments. Surface HSP70 expressions were normalized to untreated control and are shown as $x$-fold induction. (c) Cytoplasmatic and surface HSP70 expression in BCP-ALL cases $(P<0.01)$. (d) Correlation of CD99 at diagnosis with induced $(s)(P 1<0.02)$ and $(c y)(P 2<0.05)$ HSP70 $(3 \mathrm{~h})$ is shown. Primary BCP-ALL samples $(n=21)$ were incubated with DN16 mAb for $3 \mathrm{~h}$, induced (s) and (cy) HSP70 were detected with anti-HSP70 Ab. (e) Representative HSP70 staining of primary patient BCP-ALL sample: flow cytometric analysis of HSP70 expression on leukemia cells incubated for $3 \mathrm{~h}$ with DN16 mAbs (red line), MOPC1 (blue line) or isotype control (black dashed line). HSP70 was detected either with HSP70FITC from 'Stressgen' (HSP70*) or with unconjugated HSP70 Ab followed by secondary step antibody conjugated with PE (HSP70**) 
There was no difference between BCP-ALL cytogenetic groups in terms of (cy)HSP70 or (s)HSP70 expression levels at diagnosis. Observed predominance of (cy)HSP70 over (s)HSP70 within BCP-ALL blasts suggests the possible reason for resistance of them to NK-mediated cell death because cytoplasmic HSP70 has the anti-apoptotic role. ${ }^{8,20}$ CD99 ligation by DN16 antibody induced rapid (within $3 \mathrm{~h}$ ) upregulation of (s)HSP70 and (cy)HSP70 (Figure 1d), and induction of both HSP70 forms clearly correlated with each other $(P<0.01 ; n=21)$ (data not shown). The level of CD99induced (cy)HSP70 (at $3 \mathrm{~h}$ ) strongly depended on (cy)HSP70 at diagnosis $(P<0.001 ; n=21)$. Expression level of CD99 on the cell surface determined both the level of CD99-induced (s)HSP70 $(P 1<0.02 ; n=21)$ and the level of (cy)HSP70 $(P 2<0.05 ; n=21)$ at the same time point (Figure $1 \mathrm{~d})$. To confirm the data obtained with HSP70 antibody from Santa Cruz, which may detect both inducible HSP70 and constitutive HSC70, we used directly conjugated HSP70-FITC (fluorescein isothiocyanate) antibody from Stressgen, specifically designed against inducible HSP70, and they also recognized CD99induced (s)HSP70 (Figure 1e).

In ET cell lines, SKES-1 and A673 CD99 only slightly upregulated HSP70 for a short period of time $(3 \mathrm{~h})$. More prominent was the response of RDES cells, but it was unstable too (Supplementary Figure 1).

CD99 ligation enhances HSP70 transcription in ALL cells. Since in all investigated cell types HSP70 was upregulated both in cytoplasm and on the cells surface, we assumed that CD99 does not induce externalization but rather protein production of HSP70. To examine the nature of CD99-induced HSP70 modulation, RT-PCR for constitutive hsc70 and inducible hsp70 was performed. Treatment of ALL cells (Reh, Jurkat) with DN16 led to increased production of hsp70 but not hsc70 mRNA (Figure 2a). Obtained data suggest that in ALL cells CD99 triggers hsp70 mRNA expression, implying that this mechanism is responsible for accumulation of HSP70 protein in the cytoplasm and on the cell surface.

The effect of CD99 ligation on HSP70 protein expression in Reh and Jurkat cells is shown in Figure $2 \mathrm{~b}$. Western blot analysis revealed strong upregulation of HSP70 in $3 \mathrm{~h}$ of incubation with anti-CD99.

In ET cell lines, TC252 and SKNMC CD99 ligation did not enhance levels of neither hsc70 nor hsp70 mRNA (Figure 2c). Even more, hsp70 mRNA was downregulated in SKNMC cells. These results corresponded to FACS data with no marked changes in HSP70 expression level in TC252 cells and slight downregulation of HSP70 on SKNMC cells (Figure 2d).

NK92-mediated cytotoxicity against ALL cells is enhanced by CD99 ligation. To examine whether NK-cell activity could be augmented by CD99-induced upregulation of (s)HSP70, we performed cytotoxicity assays with ALL and ET cell lines (4-5 assays for each cell line). As effector cells we used the CD16 ${ }^{\text {neg }}$ NK92 cell line which previously was evaluated in phase $1 / \mathrm{II}$ clinical trials for the treatment of pediatric and adolescent patients with leukemia and sarcoma. ${ }^{24,25}$ Because our results showed that CD99 is able to upregulate HSP70 on ALL, but not on examined ET cells, we suspected to see effective cytolysis after CD99 modulation only in ALL cells. Our data confirm that CD99 ligation does not increase NK92 cell cytotoxicity against TC252 or SKNMC cells (Supplementary Figure 2). In contrast, NK92mediated killing of Jurkat cells was rapidly (in $3 \mathrm{~h}$ ) increased up to 2 -fold even at the lowest $E: T$ ratio $(1: 1)$ (Figure $3 a)$. In Reh cells, this process was less effective, albeit noticeable
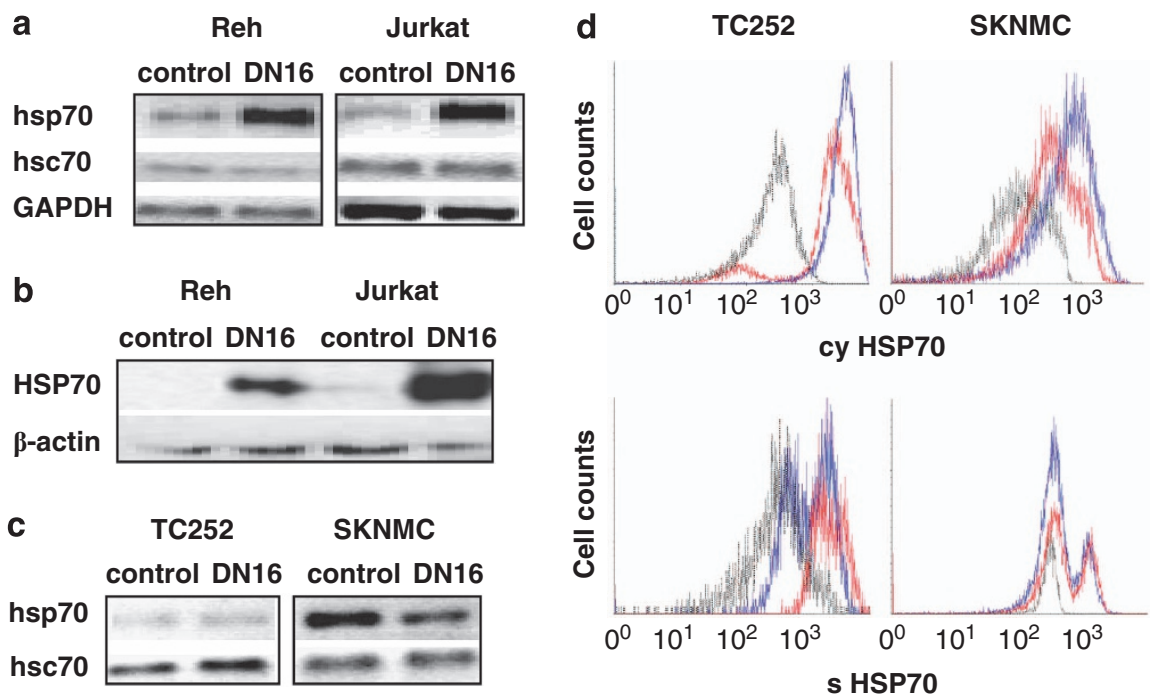

Figure 2 CD99 ligation induces HSP70 transcription in ALL but not in ET cells. (a) Treatment of ALL cells (Reh and Jurkat) with CD99 (DN16) mAb led to upregulation of hsp70 but not hsc70 mRNA expression. Representative picture of three experiments is shown (control - MOPC1-treated cells). (b) Western blot with anti-HSP70 antibody was performed after CD99 ligation by DN16 mAb in Reh and Jurkat cells. $\beta$-Actin was used as the loading control. (c) RT-PCR for constitutive hsc70 and inducible hsp70 was performed using TC252 and SKNMC cell lines. RNA input was normalized by equal number of non-apoptotic cells loaded per sample. (d) Corresponding FACS data showed no differences in HSP70 expression level in TC252 cells and slight downregulation of HSP70 on SKNMC cells (blue lines - MOPC1-treated cells, red lines - CD99 (DN16)treated cells, black dashed lines - isotype control) 
(Figure 3b). Also the three primary BCP-ALL cases tested demonstrated an increased sensitivity to NK cells upon CD99 ligation (Figure $3 \mathrm{c}$ ). This seems important because BCP-ALL cell lines as well as primary B-lineage leukemic blasts were shown previously to be rather resistant to NK cell-mediated cytotoxicity. ${ }^{22,26}$

\section{Discussion}

CD99 modulation in different cell types often leads to divergent responses. Recruitment of distinct targets to CD99-regulated processes can be one of the possible explanations. Here, we describe a novel signaling pathway where CD99 in T- and B-leukemia cells modulates expression of HSP70 on mRNA and protein levels. As we realized that CD99 upregulated HSP70 protein both in cytoplasm and on the cell surface, we hypothesized that CD99-induced surface HSP70 can be involved in stimulation of cytolytic NK cells activity. Cytotoxicity assays confirmed this idea, showing death of targeted ALL cells which correlated with the induction of HSP70.

Functional engagement of targets, such as specific surface antigens, on tumor cells is a mechanism to increase the lytic efficacy of cytotoxic cells against such tumors. ${ }^{24}$ We consider CD99 to be a strong candidate for this role. Members of the HSP70 family, being the recognition site for NK cells, are in the focus of another approach, due to their capacity to activate immunity. ${ }^{25}$ Ligation of CD99 to increase HSP70 on the surface of targeted cells could be a lucky combination of these two anticancer therapeutic approaches.

Lack of NK-cell activation is proposed to be a general mechanism of BCP leukemic cell resistance. ${ }^{26}$ Since HSP70 serves as a recognition site ${ }^{27}$ and an activating molecule m $^{21,23}$ for killer cells, NK cell-mediated cytolysis may correlate with HSP70 expression on target cells. Our results suggest CD99 ligation on leukemia cells as effective tool to increase NK-cell activity toward targets due to upregulation of HSP70. On the other hand, according to the hypothesis of Ljunggren and Kärre ${ }^{28}$ the targets of NK cells should be devoid of MHC class 1 human leukocyte protein antigens (HLA) which otherwise inhibit NK-cell cytotoxicity. It was shown that blocking of $\mathrm{MHC}$ class 1 antigen with mAbs on leukemic B cells resulted in higher susceptibility to lysis. ${ }^{29}$ Recently, HLA1 was found to be dependent on CD99. ${ }^{30}$ These observations suggest that blocking of CD99 may increase the efficacy of target cell lysis by NK cells due to HLA class I downregulation. However, NK92 cells used in our study are devoid of almost all known killer cell immunoglobulin-like receptors (KIRs), whose major ligands are $\mathrm{MHC}$ I molecules. ${ }^{22,24,26}$ Hence, our data show that even in the absence of $\mathrm{MHC1}$-dependent recognition, CD99 ligation augments NK cell-mediated cytotoxicity. Moreover, the NK92 cell line is CD16-negative cells and therefore is an ideal system to measure antibody-dependent cellular cytotoxicity. ${ }^{22}$

Intriguingly, CD99-induced modulation of HSP70 on ET cells had profiles different from that on leukemia cells. Our experiments with SKNMC and TC252 cell lines demonstrated
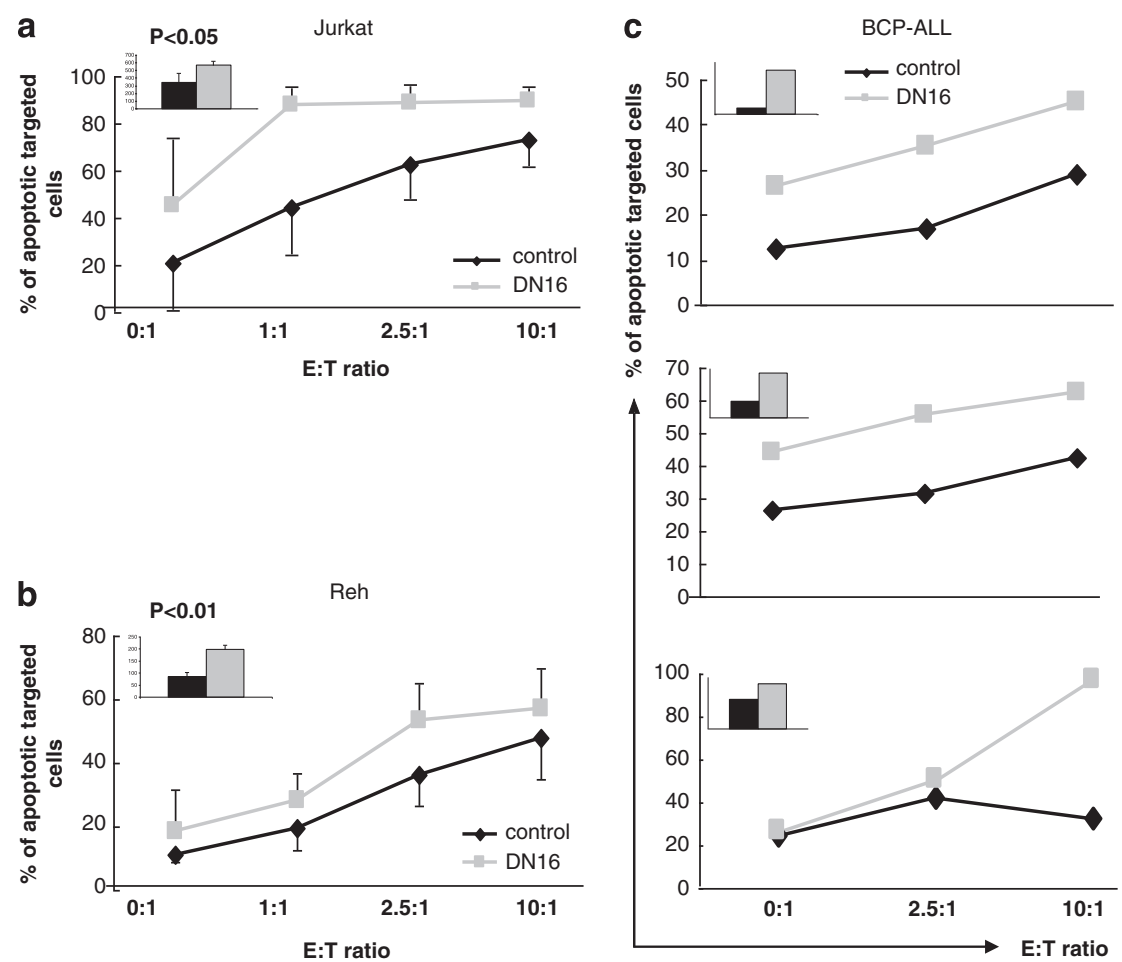

Figure 3 CD99 ligation enhances NK92-mediated cytotoxicity against ALL but not ET cells. Cytotoxicity assays with ALL and ET cells (4-5 for each cell line) were performed with NK92 cells as effectors. Annexin V and 7AAD were used to detect lysed targeted cells. CD99 induced HSP70 (insets in the graphics) and in parallel increased NK92 cytotoxicity against Jurkat (a) and Reh (b) (3 h of co-incubation) cells. (c) Cytotoxicity of NK92 cells against primary BCP-ALL (three independent ETV6/RUNX1-positive cases are shown) 
that neither HSP70 on these cells was upregulated by CD99, nor susceptibility of CD99-treated cells to NK92 was increased over the control. While this implies that HSP70 does not have a target role in ET cells, some ET cell lines were recently shown to be susceptible to NK cells due to expression of ligands for the activating NK-cell receptors NKG2D and DNAM-1. ${ }^{31}$

In conclusion, our study reveals for the first time upregulation of HSP70 in B and T leukemias upon CD99 ligation: incubation of ALL cells with CD99 antibody induced fast, stable and significant production of HSP70. RT-PCR analysis showed that this effect was initiated on the mRNA level. We demonstrated an association between CD99-induced surface HSP70 modulation and NK-cell activity against leukemic cells lines and primary BCP-ALL. In addition, we have found that 10 times lower concentration of CD99 antibody (DN16, $1 \mu \mathrm{g} / \mathrm{ml}$ ) also successfully upregulates surface HSP70 without induction of antibody-mediated cell death (Supplementary Figure 3). It may be important to spare non-leukemic, normal immature BCP with high level of CD99 from the direct killing by CD99 antibodies. ${ }^{4}$

Together, obtained results should be anticipated as a new view on CD99 functions because cooperation of CD99 with a member of heat-shock proteins family - most conserved proteins in living organisms - allowed expecting even more diverse CD99 roles than was assumed before. We hope that our data may have a vital clinical impact on future CD99targeted therapies.

\section{Materials and Methods}

Cells. BCP-ALLs were diagnosed centrally according to standard clinical criteria, genetic and immunophenotype analysis. Patients were recruited to the study which was approved by the institutional review board and informed consent was obtained from parents or legal guardians for sampling procedures and subsequent laboratory investigations. Primary leukemia mononucleated cells (MNC) from patients at diagnosis as well as normal MNC were purified from the BM samples by Ficoll-Paque (GE Healthcare, Vienna, Austria) density gradient centrifugation. Cells were either maintained in serum-free AIM V medium (Invitrogen, Lofer, Austria) or cryopreserved in liquid nitrogen. Before experiments, cells were washed with AIM V supplemented with $10 \%$ FBS, resuspended in AIM V with $1 \%$ FBS and used for cytotoxicity assay. ALL cell lines (Reh, ETV6/RUNX1-positive BCP-ALL, and Jurkat, T-ALL) (obtained from DSMZ, Braunschweig, Germany) and ET cell lines (TC252, SKNMC, RDES, A673 and SKES1, the kind gift of Prof. $\mathrm{H}$ Kovar, cultured in house) were maintained in RPMI medium (Invitrogen) with supplements and before the experiments were washed and switched to AIM V medium. NK92 cells (from DSMZ) were maintained in X-VIVO 10 medium (Biowhittaker, MD, USA) containing $20 \%$ fetal bovine serum 'Gold' (PAA Laboratories, Pasching, Austria), $200 \mathrm{U} / \mathrm{ml}$ interleukin-2 (PeproTech, Vienna, Austria) and supplements. All cell lines were monitored in regular intervals by PCR to exclude mycoplasma infection.

Antibodies, materials and reagents. The following anti-CD99 mAbs were used for CD99 ligation: DN16 from Dinona (Seoul, South Korea), hec2 - a gift from Dr. A Müller (Weill Medical College, Cornell University, New York, NY, USA) and 0662 - a gift from Dr. A Bernard (Institute National de la Santé et de la Recherche Médicale, Nice, France). MOPC-21 (IgG1 irrelevant mAb) was from Sigma (St. Louis, MO, USA), CD10 and CD19 - from Abcam (Cambridge, UK). CD99 expression analysis was done with FITC-conjugated DN16 mAb (ABD Serotec, Düsseldorf, Germany), as well as in selected experiments with phycoerythrin (PE)-conjugated 3B2 mAb (Caltag, Hamburg, Germany). HSP70 (W27) un-conjugated and PE-conjugated Abs were from Santa Cruz Biotechnology (Santa Cruz, CA, USA), HSP70-FITC - from Stressgen (Victoria, BC, Canada). A secondary PE-conjugated goat anti-mouse IgG was from Dako (Glostrup, Denmark). MOPC-21 was added before staining with secondary lgG-PE to reduce background binding. FITC- or APC-conjugated Annexin V and 7AAD (amino-actinomycin D) were from BD Biosciences (San Jose, CA, USA). All cell culture reagents were purchased from Invitrogen.

HSP70 detection. Cells were cultured at $2 \times 10^{5} / 200 \mu \mathrm{l}$ in 96-well tissue culture plates (Nunc, Roskilde, Denmark) or in 25-ml culture flasks (Nunc, Vienna, Austria). The cells were treated with azide-free anti-CD99 mAbs (DN16) at a final concentration of $10 \mu \mathrm{g} / \mathrm{ml}$ (in detected experiments - with $1 \mu \mathrm{g} / \mathrm{ml}$ ). As a negative control, the isotype-matched control antibody MOPC-21 was used. Plates were incubated at $37^{\circ} \mathrm{C}, 5 \% \mathrm{CO}_{2}$ in a humidified incubator up to 7 days. Immunophenotyping and apoptosis assessment were processed at indicated time points.

Detection and quantification of cells was done by the flow cytometric analysis of 7AAD/Annexin V-labeled cells. The assay was performed according to manufacturer's instructions. Briefly, cells were collected from culture, suspended in $100 \mu$ l Annexin V binding buffer (10 mM HEPES, pH 7.4, $2.5 \mathrm{mM} \mathrm{CaCl}_{2}, 140 \mathrm{mM}$ $\mathrm{NaCl}$ ) containing $1 \mu \mathrm{g} / \mathrm{ml}$ Annexin V. After 15 min incubation in the dark at room temperature, 7AAD ( $1 \mu$ l/tube) was added and dead cells were quantified on the flow cytometer. HSP70 expression was measured on the gated alive, non-apoptotic $\left(7 A A D^{-}\right.$Annexin $\left.V^{-}\right)$cells. Cytoplasmatic staining for HSP70 analysis was performed with the fixation/permeabilization kit from Caltag according to manufacturer's recommendations. Afterwards, the cells were washed and analyzed on the FACS Calibur (BD).

RNA isolation and RT-PCR. Total RNA was isolated using RNeasy Micro Kit (Qiagen, Hilden, Germany). Single-strand cDNA was synthesized and HSP70 was PCR amplified using the following specific primers (all from Eurofins MWG Operon, Ebersberg, Germany): hsc70 forward 5'-TGTGGCTTCCTTCGTTATTGG$3^{\prime}$ and reverse $5^{\prime}$-GCCAGCATCATTCACCACCAT-3'; ${ }^{\prime}$ ' hsp70 forward: $5^{\prime}$-AGAGCCGAGCCGACAGAG-3' and reverse: $5^{\prime}$-CACCTTGCCGTGTTGGAA-3'; GAPDH forward: $5^{\prime}$-CCACTCCTCCACCTTGAC-3' and reverse: $5^{\prime}$-ACCCTGTTGCTGTAGCCA- $3^{\prime} .^{33}$ PCR products were separated on $2 \%$ agarose gel and visualized with ethidium bromide.

Western blot analysis. Total proteins were fractionated by $10 \%$ SDSPAGE, transferred onto nitrocellulose membrane (Whatman, Dassel, Germany) and processed according to standard procedures. Membranes were probed with anti-HSP70 (Santa Cruz) and anti- $\beta$-actin antibody (Abcam).

Cytotoxicity assay. As effector cells we used NK92 cell line with confirmed high cytotoxic activity. ${ }^{22}$ Cytotoxic activity was evaluated by FACS analysis similar to previously described method ${ }^{26}$ but with some modifications. Briefly, target cells (minimum $10 \times 10^{3}$ ) were incubated with or without DN16 mAb for $3 \mathrm{~h}$ (ET cells for $24 \mathrm{~h}$ ) and then effector cells were added to get effector-to-target ratios $(E: T)$ of $1: 1,2.5: 1,5: 1,10: 1$ and $20: 1$. ALL and ET cells were then incubated with effector cells for 3 and $24 \mathrm{~h}$. Afterwards, cell mixture was harvested from 96 U-bottom plates and stained with Annexin V, 7AAD and cell type-specific markers. Dead target cells were identified as 7AAD + and Annexin $\mathrm{V}^{+}$, and excluded from further analysis. Target cells were identified as follows: Reh as CD10 ${ }^{+}$, Jurkat as $\mathrm{CD}^{+}, \mathrm{TC}^{2} 22$ and SKNMC as $\mathrm{CD}_{6}{ }^{-}$. Control target cells were incubated without effector cells and without DN16.

Statistical methods. All data are shown as mean \pm standard error of the mean (S.E.M.). Statistical significance of the difference in mean cell recovery/ apoptosis between targeted and control cells was determined by the Wilcoxon signed rank test. Correlation analysis was performed by the Spearman test. The Mann-Whitney test was used to assess the differences in proteins levels.

\section{Conflict of Interest}

The authors declare no conflict of interest.

Acknowledgements. We are thankful to Dr. H Kovar for critical reading of our manuscript. We thank M Zavadil for secretarial assistant. This work was supported by ÖNB Jubiläumsfond (grant No. 13081).

\section{Author contributions}

$\mathrm{ZH}$ designed and performed the experiments, analyzed the data, generated the figures and wrote the manuscript. MD was responsible for the overall study support and for editing the manuscript. 
1. Kovar H, Dworzak M, Strehl S, Schnell E, Ambros IM, Ambros PF et al. Overexpression of the pseudoautosomal gene MIC2 in Ewing sarcoma and peripheral primitive neuroectodermal tumor. Oncogene 1990; 5: 1067-1070.

2. Dworzak MN, Fritsch G, Buchinger P, Fleischer C, Printz D, Zellner A et al. Flow cytometric assessment of human MIC 2 expression in bone marrow, thymus, and peripheral blood. Blood 1994; 83: 415-425.

3. Dworzak MN, Fritsch G, Fleischer C, Printz D, Fröschl G, Buchinger P et al. CD99 (MIC2) expression in paediatric $B$-lineage leukaemia/lymphoma reflects maturation-associated patterns of normal B-lymphopoiesis. Br J Haemat 1999; 105: 690-695.

4. Husak Z, Printz D, Schumich A, Pötschger U, Dworzak MN. Death induction by CD99 ligation in TEL/AML1-positive acute lymphoblastic leukemia and normal B-cell precursors. J Leuk Biol 2010; 88: 405-412

5. Hahn JH, Kim MK, Choi EY, Kim SH, Sohn HW, Ham DI et al. CD99 (MIC2) regulates the LFA-1/ICAM-1-mediated adhesion of lymphocytes, and its gene encodes both positive and negative regulators of cellular adhesion. J Immunol 1997; 159: 2250-2258.

6. Alberti I, Bernard G, Rouquette-Jazdanian AK, Pelassy C, Pourtein M, Aussel C et al. CD99 isoform expression dictates T-cell functional outcomes. FASEB J 2002; 16 : 1946-1948.

7. Schenkel AR, Mamdouh Z, Chen X, Liebman RM, Muller WA. CD99 plays a major role in the migration of monocytes through endothelial junctions. Nat Immunol 2002; 3 : 143-150.

8. Jaattela M. Heat shock proteins as cellular lifeguards. Ann Med 1999; 31: 261-271.

9. Wehner PS, Nielsen B, Hokland M. Expression levels of HSC70 and HSP60 are developmentally regulated during B-cell maturation and not associated to childhood c-ALL at presentation or relapse. Eur J Haematol 2003; 71: 100-108.

10. Hantschel IM, Pfister K, Jordan A, Scholz R, Andersen R, Schmitz G et al. Hsp70 plasma membrane expression on primary tumor biopsy material and bone marrow of leukemic patients. Cell Stress Chaperones 2000; 5: 438-442.

11. Verrills NM, Liem NL, Liaw TY, Hood BD, Lock RB, Kavallaris M. Proteomic analysis reveals a novel role for the actin cytoskeleton in vincristine resistant childhood leukemia an in vivo study. Proteomics 2006; 6: 1681-1694.

12. Cerisano V, Aalto Y, Perdichizzi S, Bernard G, Manara MC, Benini S et al. Molecular mechanisms of CD99-induced caspase-independent cell death and cell-cell adhesion in Ewing's sarcoma cells: actin and zyxin as key intracellular mediators. Oncogene 2004; 23: 5664-5674.

13. Wells AD, Rai SK, Salvato MS, Band H, Malkovsky M. Hsp72-mediated augmentation of MHC class I surface expression and endogenous antigen presentation. Int Immunol 1998; 10: $609-617$.

14. Sohn HW, Shin YK, Lee IS, Bae YM, Suh YH, Kim MK et al. CD99 regulated the transport of MHC class I molecules from the Golgi complex to the cell surface. J Immunol 2001; 166: 787-794.

15. Yoon SS, Jung KI, Choi YL, Choi EY, Lee IS, Park SH et al. Engagement of CD99 triggers the exocytic transport of ganglioside GM1 and the reorganization of actin cytoskeleton. FEBS Lett 2003; 540: 217-222.

16. Dall'Era MA, Oudes A, Martin DB, Liu AY. Hsp27 and Hsp70 interact with CD10 in C4-2 prostate cancer cells. Prostate 2007; 67: 714-721.

17. Wang R, Kovalchin JT, Muhlenkamp P, Chandawarkar RY. Exogenous heat shock protein 70 binds macrophage lipid raft microdomain and stimulates phagocytosis, processing and MHC-II presentation of antigens. Blood 2006; 107: 1636-1642.
18. Diakos C, Krapf G, Gerner C, Inthal A, Lemberger C, Ban J et al. RNAi-mediated silencing of TEL/AML1 reveals a heat-shock protein- and surviving-dependent mechanism for survival. Blood 2006; 109: 2607-2609.

19. Wykoff CC, Pugh CW, Maxwell PH, Harris AL, Ratcliff PJ. Identification of novel hypoxia dependent and independent target genes of the von Hippel-Lindau (VHL) tumour suppressor by mRNA differential expression profiling. Oncogene 2000; 19: 6297-6305.

20. Creagh EM, Sheehan D, Gotter TG. Heat shock proteins - modulators of apoptosis in tumour cells. Leukemia 2000; 14: 1161-1173.

21. Elsner L, Muppala V, Gehrmann M, Lozano J, Malzahn D, Bickeböller $\mathrm{H}$ et al. The heat shock protein HSP70 promotes mouse NK cell activity against tumors that express inducible NKG2D ligands. J Immunol 2007; 179: 5523-5533.

22. Yan Y, Steinherz P, Klingemann HG, Dennig D, Childs BH, McGuirk J et al. Antileukemia activity of a natural killer cell line against human leukemias. Clin Cancer Res 1998; 4: 2859-2868.

23. Creagh EM, Cotter TG. Selective protection by hsp70 against cytotoxic drug, -but not Fas-induced T-cell apoptosis. Immunology 1999; 97: 37-44.

24. Suck G. Novel approaches using natural killer cells in cancer therapy. Semin Cancer Biol 2006; 16: 412-418.

25. Srivastava PK. Immunotherapy for human cancer using heat shock protein-peptide complexes. Curr Oncol Rep 2005; 7: 104-108.

26. Romanski A, Bug G, Becker S, Kampfmann M, Seifried E, Hoelzer D et al. Mechanisms of resistance to natural killer cell-mediated cytotoxicity in acute lymphoblastic leukemia. Exp Hematol 2005; 33: 344-352.

27. Multhoff G, Botzler C, Jennen L, Schmidt J, Ellwart J, Issels R. Heat schock protein 72 on tumor cells: a recognition structure for natural killer cells. J Immunol 1997; 158: 4341-4350.

28. Ljunggren HG, Kärre K. In search of the 'missing self': MHC molecules and NK cell recognition. Immunol Today 1990; 11: 237-244.

29. Palucka KA, Reizenstein P, Öst A, Pozwitmacdonald A. Blocking of MHC class I antigens on leukemic B-cells enhances their conjugate formation with cytotoxic lymphocytes and their susceptibility to lysis. Leuk Lymphoma 1998; 28: 573-581.

30. Bremond A, Meynet O, Mahiddine K, Coito S, Tichet M, Scotlandi K et al. Regulation of HLA1 class I surface expression requires CD99 and p230/golgin-245 interaction. Blood 2009; 113: 347-357.

31. Verhoeven DH, de Hooge AS, Mooiman EC, Santos SJ, ten Dam MM, Gelderblom H et al. NK cells recognize and lyse Ewing sarcoma cells through NKG2D and DNAM-1 receptor dependent pathways. Mol Immunol 2008; 45: 3917-3925.

32. Kim YH, Park EJ, Han ST, Park JW, Kwon TK. Arsenic trioxide induces Hsp70 expression via reactive oxygen species and JNK pathway in MDA231 cells. Life Sci 2005; 77 : 2783-2793.

33. Westerheide SD, Anckar J, Stevens SM Jr, Sistonen L, Morimoto RI. Stress-inducible regulation of heat shock factor 1 by the deacetylase SIRT1. Science 2009; 323 1063-1066.

Cell Death and Disease is an open-access journal published by Nature Publishing Group. This work is licensed under the Creative Commons Attribution-NonCommercial-No Derivative Works 3.0 Unported License. To view a copy of this license, visit http://creativecommons.org/licenses/by-nc-nd/3.0/

Supplementary Information accompanies the paper on Cell Death and Disease website (http://www.nature.com/cddis) 